\title{
REFLEXIONES ACERCA DEL ROL DEL ESTADO EN LAS CONTROVERSIAS DE SALUD: GARANTE PRIMARIO O SUBSIDIARIO
}

\author{
REFLECTIONS ON THE ROLE OF THE STATE \\ IN HEALTH CONTROVERSIES: PRIMARY OR \\ SUBSIDIARY GUARANTOR
}

\section{María Alejandra Asencio ${ }^{1}$ - Fedra Carla Gatti²}

DOI: https://doi.org/10.37767/2591-3476(2021)13

\section{Comentario a}

Padilla, Laura Rosario c/ Hospital de General Roca y otros s/ Amparo -apelación

Corte Suprema de Justicia de la Nación

\section{Disponible en}

https://bit.ly/3BvIREL

\section{RESUMEN:}

En el presente artículo se analiza la decisión adoptada por la Corte Suprema de Justicia de la Nación en una acción de amparo de salud, desde una perspectiva de la protección integral de la discapacidad y del enfoque protectorio de los derechos humanos. Asimismo, se indaga respecto del carácter principal o subsidiario de la responsabilidad del Estado en su rol de garante del excelso derecho a la salud de las personas discapacitadas.

\begin{abstract}
In this work, the adopted decision of the Supreme Court of Justice of the Nation in a health protection action is analyzed. The comprehensive protection of disability and the protective approach to human rights is used as a comprehensive context. Likewise, the principal or subsidiary nature of the responsibility of the State, in its role as guarantor of the right to health of disabled persons, is investigated.
\end{abstract}

\footnotetext{
1 Abogada (UNC); Egresada sobresaliente. Diplomada en Derecho Procesal Civil (UBP); Se desempeña profesionalmente en la Cámara Contencioso Administrativa de Segunda Nominación de Provincia de Córdoba. Mail: aleasencio@gmail.com. ORCID iD: https://orcid.org/0000-0001-9147-2609.

2 Abogada (UNC); Egresada sobresaliente. Adscripta a la Cátedra de Derecho Procesal Administrativo (UNC); Maestrando en Gerencia y Administración en Servicios de Salud (Escuela de Salud Pública - UNC). Se desempeña profesionalmente en la Secretaría Electoral y de Competencia Originaria del Tribunal Superior de Justicia de Córdoba. Mail: fedragatti@gmail.com. ORCID iD: https://orcid.org/0000-0002-4382-5948
} 
PALABRAS CLAVE: Derecho a la salud - Protección integral de la Discapacidad- - Responsabilidad del Estado - Carácter principal o subsidiario - Extrema vulnerabilidad .

KEY WORDS: Right to health - Comprehensive Disability Protection - State responsibility The principal or subsidiary character - Extreme vulnerability.

\section{Introducción}

El propósito de estas consideraciones es efectuar un análisis del caso judicial que nos convoca desde una perspectiva de la garantía constitucional del debido proceso en el marco de la acción de amparo formulada por la actora, paciente en situación de discapacidad, a fin de obtener de parte del Estado Provincial de Río Negro y de los codemandados, Ministerio de Salud Pública de la Provincia de Río Negro y del Hospital de General Roca, la cobertura integral de ciertas prestaciones en resguardo de su derecho de salud y en base a la existencia de un acuerdo previo celebrado con la Provincia.

La metodología interpretativa seguida en el caso judicial por el Máximo Tribunal de la Nación invita a reflexionar acerca de dos conceptos fundamentales: la protección integral de la discapacidad y la obligación del Estado en el marco de la legislación de protección integral de la discapacidad.

Tal como lo sostendremos, se trata de dos conceptos que, en base a un razonable equilibrio, conviven en una dialéctica destinada a la tutela preferente del derecho a la salud de las personas con discapacidad.

Finalmente, desde un enfoque de los derechos humanos y en base a la incontrastable realidad de demandas crecientes de cobertura de salud en casos complejos, efectuaremos una especial consideración sobre la obligación del Estado como garante del derecho a la salud, delineando los límites de su responsabilidad y el carácter en el cual resulta comprometido en este tipo especial de procesos.

\section{El caso bajo examen}

El Superior Tribunal de Justicia de la Provincia de Río Negro revocó el decisorio de la primera instancia e hizo lugar, por vía de apelación, a la petición de la actora acerca de la cobertura total de las prestaciones médicas que aquella requiriese por su situación de discapacidad, sin perjuicio de reclamar a la obra social.

A su tiempo, la Jueza de Primer Grado había rechazado la acción de amparo planteada al considerar que, conforme lo acreditado en autos, no se encontraba violentado el derecho a la salud, debiendo recurrirse en primer término a la obra social a efectos de obtener la cobertura indicada, sin perjuicio de que el sistema público pudiese amparar a la actora en caso de urgencia con la opción de repetir a la obra social.

A los fines de fundamentar su resolución, el Superior Tribunal valoró la realidad económica de la actora quien, como afiliada, no habría podido afrontar el costo de los coseguros de la Obra Social OSDOP, y consideró especialmente la adhesión del Estado Provincial a la ley 24.901, al afirmar que tanto esta ley como la normativa prevista por la ley 22.431 "ponen a cargo del Estado el sistema de prestaciones básicas para discapacitados". 
De tal modo que, el Tribunal de Apelación pese a enfatizar sobre la obligación prevista por el art. $2^{\circ}$ de aquella norma en cuanto dispone "Las obras sociales, ... tendrán a su cargo, con carácter obligatorio, la cobertura total de las prestaciones básicas enunciadas en la ley, que necesiten las personas con discapacidad afiliadas a las mismas", concluyó que el hecho de que la peticionaria fuese afiliada a una obra social era insuficiente para liberar al Estado de las obligaciones que pesaban sobre él por integrar el sistema de salud.

Contra ese fallo, la demandada interpuso recurso extraordinario federal en base a la causal de arbitrariedad pues, desde su posición, se habrían soslayado cuestiones de hecho fundamentales toda vez que no se acreditó en el expediente que la actora se encontrase en situación de urgencia ni mucho menos que hubiese quedado sin atención médica.

Adicionó como agravio federal el argumento de que la decisión impugnada viola el derecho federal, al sustentarse en una interpretación que desconoce la normativa aplicable al caso; esto es, que el sistema de salud se estructura en base a las leyes nacionales 23.660 (art. 3) y 23.661 (arts. 1 y 2); art. 59 de la Constitución de la Provincia de Río Negro y ley R 2570 (art. 1). En consecuencia, a su entender, la atención médica de la actora debía canalizarse, en principio, a través de la Obra Social respecto de la cual la amparista era afiliada y subsidiariamente, a través del Estado en su rol de garante de la salud, lo cual fue soslayado por el Superior Tribunal de la Provincia.

En esta tesitura, el Estado Provincial demandado insistió en que el fallo generaba a su cargo obligaciones inexistentes al tiempo que lo obligaba a utilizar fondos públicos para financiar a la Obra Social no demandada, ocasionándole una ruptura de su equilibrio financiero público local e incurriendo en una verdadera gravedad institucional.

Por su parte, en su decisión, el Máximo Tribunal de la Nación, consideró que el derecho de defensa en juicio y del debido proceso consagrados por la norma constitucional, constituían materia federal suficiente y en consecuencia, invalidó el pronunciamiento de lo actuado y decidido en el marco del proceso por el Tribunal ad quo.

De los términos reseñados del caso judicial, y tal como fuese anunciado, la interpretación de la Corte nos orienta hacia una primera categoría de análisis: la obligación del Estado como responsable y garante del derecho a la salud. A partir de ello, amplía nuestra indagación respecto del carácter principal o subsidiario de la responsabilidad del Estado en su rol de garante del excelso derecho a la salud de las personas discapacitadas.

\section{Derecho a la salud. Concepto. La obligación del Estado en el marco de la protec- ción integral de la discapacidad}

Del breve repaso de la jurisprudencia de la Corte Suprema de Justicia de la Nación, se destacan las siguientes nociones básicas: "El derecho a la salud, máxime cuando se trata de enfermedades graves, está íntimamente relacionado con el derecho a la vida, siendo éste el primer derecho de la persona humana que resulta reconocido y garantizado por la Constitución Nacional; el hombre es el eje y centro de todo el sistema jurídico y en tanto fin en sí mismo -más allá de su naturaleza trascendente- su persona es inviolable y constituye un valor fundamental, con respecto al cual los restantes valores tienen siempre carácter instrumental" (329:4918).

“El derecho a la vida, más que un derecho no enumerado en los términos del art. 33 de 
la Ley Fundamental, es una prerrogativa implícita, ya que el ejercicio de los derechos reconocidos expresamente requiere necesariamente de él y, a su vez, el derecho a la salud está íntimamente relacionado con el primero y con el principio de autonomía personal, desde que un individuo gravemente enfermo no está en condiciones de optar libremente por su propio plan de vida" (329:4918)

Por su parte, la Constitución de la Organización Mundial de la Salud consagra que “La salud es un estado de completo bienestar físico, mental y social, y no solamente la ausencia de afecciones o enfermedades. El goce del grado máximo de salud que se pueda lograr es uno de los derechos fundamentales de todo ser humano sin distinción de raza, religión, ideología política o condición económica o social... Los gobiernos tienen responsabilidad en la salud de sus pueblos, la cual sólo puede ser cumplida mediante la adopción de medidas sanitarias y sociales adecuadas". (OMS, 2017).

A su turno, la Corte Interamericana de Derechos Humanos define el derecho a la salud como "Un derecho humano fundamental e indispensable para el ejercicio adecuado de los demás derechos humanos, y que todo ser humano tiene derecho al disfrute del más alto nivel posible de salud que le permita vivir dignamente, entendida la salud no sólo como la ausencia de afecciones o enfermedades, sino también como un estado completo de bienestar físico, mental y social, derivado de un estilo de vida que permita alcanzar a las personas un balance integral" (Corte IDH. Caso Poblete Vilches y otros Vs. Chile. Fondo, Reparaciones y Costas. Sentencia de 8 de marzo de 2018. Serie C No. 349, § 118; Caso Cuscul Pivaral y otros Vs. Guatemala. Excepción Preliminar, Fondo, Reparaciones y Costas. Sentencia de 23 de agosto de 2018. Serie C No. 359, § 105; Caso Hernández Vs. Argentina. Excepción Preliminar, Fondo, Reparaciones y Costas. Sentencia de 22 de noviembre de 2019. Serie C No. 395, § 76).

En virtud de lo dispuesto por los tratados internacionales, que en nuestro sistema normativo gozan de jerarquía constitucional (art. 75, inc. 22, de la C.N.), se reafirma el derecho a la preservación de la salud, y como su corolario, la obligación impostergable del Estado en miras de garantizar este derecho con acciones positivas, sin perjuicio de las obligaciones que deban asumir en su cumplimiento las jurisdicciones locales, las obras sociales o las entidades de la llamada medicina prepaga. En este sentido, se ha expedido el Máximo Tribunal en innumerables precedentes jurisprudenciales (CSJN, Fallos 323:3229 in re "Campodónico de Beviacqua"; 321:1684; 323:1339, mayoría y votos concurrentes y dictamen del señor Procurador General de la Nación a cuyos fundamentos se remiten)

Expresamente, el art. 75, inc. 23 de la C.N. consagra esta obligación en cuanto dispone que corresponde al Congreso Nacional "legislar y promover medidas de acción positiva que garanticen la igualdad real de oportunidades y de trato, y el pleno goce y ejercicio de los derechos reconocidos por esta Constitución y por los tratados internacionales vigentes sobre derechos humanos, en particular respecto de los niños, las mujeres, los ancianos y las personas con discapacidad".

Lo afirmado se confirma en materia de regulación de la discapacidad, pues en consonancia con las previsiones sobre el Sistema de protección integral de los discapacitados de la ley nacional 22.431 (B.O. 16/03/1981), la ley nacional 24.901 (B.O. 05/12/1997), bajo el título de Sistema de prestaciones básicas en habilitación y rehabilitación integral a favor de las personas con discapacidad, instituyó "un sistema de prestaciones básicas de atención in- 
tegral a favor de las personas con discapacidad, contemplando acciones de prevención, asistencia, promoción y protección, con el objeto de brindarles una cobertura integral a sus necesidades y requerimientos" (art. 1).

Asimismo, dispuso que "Las obras sociales, comprendiendo por tal concepto las entidades enunciadas en el artículo $1^{\circ}$ de la ley 23.660 , tendrán a su cargo con carácter obligatorio, la cobertura total de las prestaciones básicas enunciadas en la presente ley, que necesiten las personas con discapacidad afiliadas a las mismas" (art. 2).

A renglón seguido, el legislador reconoció la trascendente misión del Estado en materia de protección integral de la discapacidad, modificando el anterior régimen del art. 4, primer párrafo, de la Ley 22.431, al disponer que "El Estado, a través de sus organismos, prestará a las personas con discapacidad no incluidas dentro del sistema de las obras sociales, en la medida que aquellas o las personas de quienes dependan no puedan afrontarlas, los siguientes servicios." (art. 3). Luego, el art. 4 de la ley 24.901 dejó establecido que: "Las personas con discapacidad que carecieren de cobertura de obra social tendrán derecho al acceso a la totalidad de las prestaciones básicas comprendidas en la presente norma, a través de los organismos dependientes del Estado".

Es decir, tras la reforma introducida y del juego armónico de sus disposiciones, la cobertura de atención integral a favor de las personas con discapacidad en primer lugar está a cargo de las obras sociales y empresas de medicina pre paga, resultando entonces la obligación de su cobertura integral por el Estado sólo procedente en caso de ausencia de afiliación.

\section{El Estado como garante del derecho a la salud: el carácter primario o subsidiario de su responsabilidad.}

De todo lo relevado, resulta evidente que existe una obligación de fuente constitucional a cargo del Estado como garante del derecho a la salud. Su fundamento se encuentra, además, avalado en los compromisos internacionales asumidos por el Estado Argentino, quien deberá, a tales fines, adoptar acciones positivas, a través del diseño y gestión de políticas públicas.

En igual sentido, tal como fuere reseñado el plexo normativo conformado por las leyes 22.431 y 24.091 pone a cargo del Estado la atención y asistencia integral de la discapacidad en cuanto constituye una política pública del país.

Sentado ello, y adentrándonos en nuestro análisis nace el interrogante respecto del alcance material de la responsabilidad del Estado en materia de salud y, más específicamente, si procede en controversias de protección de la discapacidad con carácter primario o de manera subsidiaria.

En lo concerniente al fallo comentado, el Alto Cuerpo, entendió que la decisión sustentada por el Tribunal a quo resultaba dogmática, pues otorgó un tratamiento inadecuado a la controversia suscitada, al apartarse de las circunstancias del caso - amparista afiliada a una obra social no demandada en primer lugar-y de las normas aplicables a su respecto (Ley 24.901), dando a la decisión un fundamento solo aparente y, por ende, afectando la garantía constitucional de defensa en juicio del Estado Provincial demandado.

En su decisorio, la Corte Suprema no se pronunció expresamente respecto de la legitimación procesal pasiva de los demandados Estado Provincial y Ministerio de Salud de la Provincia de Río Negro, a diferencia de la postura asumida por la Jueza de Primera 
Instancia y del voto en disidencia del Dr. Sergio M. Barotto, Vocal del Superior Tribunal de Río Negro, quien al momento de resolver sobre la concesión del recurso extraordinario federal expuso: "atento a que los amparistas iniciaron la presente acción contra el Estado Provincial - Ministerio de Salud Pública, en el entendimiento de que éste era el legitimado pasivo, sujeto primario de la obligación de garantizar la salud integral, advertí (citando el precedente "Esparza", ST JRNS4, Se. 163/12) que no corresponde al Ministerio de Salud de la Provincia de Río Negro asegurar el derecho a la salud de quien posee una Obra Social obligada a dicha cobertura, contra quien efectivamente debió incoarse la acción".

Sin embargo, la Corte dejó a salvo que a la luz de la legislación aplicable al caso y del estudio de las constancias de la causa y considerando los términos en que había sido formulada la acción de amparo, el Hospital "Francisco López Lima" de General Roca, el Ministerio de Salud Pública provincial y la Provincia de Río Negro no podían ser demandados en forma directa respecto de las prestaciones de salud reclamadas.

En el caso judicial las contingencias propias de la causa y la relación jurídica existente entre la actora en su carácter de afiliada y la obra social no autorizaban su proposición como demandados directos y únicos responsables.

Más aún, en su Considerando $5^{\circ}$ la Corte Suprema hizo hincapié en el hecho de que la propia actora, al interponer la acción, no cuestionó la falta de atención de sus patologías por parte del hospital local, sino que lo que concretamente reclamó es la atención con carácter preferente por parte de especialistas y la entrega de medicamentos, anteojos y audífonos, entre otras prestaciones, a la que habría creído tener derecho, con base en el acuerdo celebrado con la Provincia mediante el que se había estipulado una indemnización por daños y perjuicios a su favor.

En ese entendimiento, en ocasión de contestar el traslado del recurso extraordinario federal la Defensora General, representante de la actora, manifestó: "el voto mayoritario (del Superior Tribunal de Río Negro) no se expidió sobre el contenido del convenio, dado que la amparista tiene derecho a ser asistida por el servicio de salud pública y el Estado Provincial se encuentra obligado a prestarlo sin necesidad que existiera aquel", argumento que fue recibido por el Alto Tribunal Provincial en la denegación del recurso en cuestión.

Es claro que, si la acción de amparo sólo resulta admisible para conjurar, evitar o remediar un acto u omisión de una autoridad pública o de particulares que conlleve una arbitrariedad o ilegalidad manifiesta (art. 43 C.N.), en este caso no se configuró, pues el convenio en el que la actora fundó su pretensión no incluyó disposición alguna sobre la atención de su salud como tampoco acreditó que hubiese requerido a su obra social las prestaciones en cuestión.

En otras palabras, el Hospital "Francisco López Lima" de General Roca, el Ministerio de Salud Pública provincial y la Provincia de Río Negro, no revestían el carácter de obligados directos y principales, como para exigir la integración de un litisconsorcio (pasivo) necesario ante la promoción de la acción de amparo por parte de la actora (afiliada).

Cobra relevancia que "Si bien se reconoce el carácter fundamental del derecho a la salud y la especial atención que merecen las personas con discapacidad, tales derechos de 
raigambre constitucional, así como los principios y garantías consagrados en la Constitución Nacional, no son absolutos sino que deben ser desplegados con arreglo a las leyes que reglamentan su ejercicio, en la forma y extensión que el Congreso, en uso de sus facultades propias, lo estime conveniente a fin de asegurar el bienestar general (arts. 14 y 28 de la Constitución Nacional), con la única condición de no ser alterados en su substancia" (CSJN, fallos 341:919)

Desde la doctrina se ha señalado la importancia de la relación jurídica sustancial que da sustento a la acción de amparo formulada por razones de salud, en cuanto ella "se debe esgrimir directamente contra la obra social puesto que el vínculo subyacente, donde se efectiviza la omisión prestataria, se entabla entre el ente público no estatal o sujeto privado y el afiliado" (Rodríguez Traversa, 2014).

\section{La especial responsabilidad del Estado en materia de controversias de salud en casos graves y complejos. El límite de la extrema vulnerabilidad}

Sentadas estas premisas básicas acerca de la responsabilidad del Estado, en cualquiera de sus esferas nacional, provincial o municipal, y habiendo ya delimitado sus contornos y su carácter subsidiario en cuestiones atinentes a la protección integral de la discapacidad conforme el marco normativo señalado, es preciso detenernos a fin de cuestionarnos si aquella impronta de subsidiariedad de la obligación del Estado transita los mismos carriles en casos de salud de mayor complejidad.

La pregunta obligada es acerca del temperamento que debería seguirse por parte de los actores judiciales y de la labor jurisdiccional en estos supuestos de entidad diversa, piénsese, por ejemplo, en las terapias innovadoras, en los medicamentos "huérfanos", breakthrough therapy, en situaciones de enfermedades raras o poco frecuentes, y en las alternativas terapéuticas de elevado costo.

Es decir, la cuestión reside en determinar si en aquellos casos donde el derecho a la salud se encuentra grave y complejamente comprometido por la gravedad de la patología, por obstáculos legales y/o reglamentarios (ley de creación de las Obras Sociales, Programa Médico Obligatorio en base a los cuales las entidades demandadas niegan la cobertura reclamada), por valladares de tipo económicos o por fallas del mercado (Tafani, R., 2010), los reclamos administrativos y judiciales de cobertura procederían conjuntamente contra las Obras Sociales o entidades de medicina prepaga y el Estado nacional, provincial o municipal; y en ese caso, el Estado resultaría responsable principal o subsidiariamente.

En otras palabras, y desde la solución judicial del caso comentado, ¿sólo en el supuesto del incumplimiento de la cobertura por parte de las obras sociales, se podría ocurrir al Estado Nacional y/o Provincial?, ¿el Estado es responsable sólo de manera subsidiaria?

Desde una primera aproximación, y en base a la lectura del fallo objeto de nuestro estudio, la respuesta positiva se impone, pues tal como lo resolvió el Máximo Tribunal la acción judicial no podía prosperar en contra de los demandados sino que, por el contrario, el tratamiento adecuado de la controversia suscitada y de las normas aplicables al caso, hubieran reparado en el hecho de que el Estado estaba obligado a brindar las prestaciones enunciadas en la ley 24.091 sólo de manera subsidiaria.

Pero más allá de la solución concretamente propiciada por la Corte en el caso judicial comentado, cuando el Estado, en cualquiera de los tres órdenes federales, es llamado a in- 
tervenir en los casos graves y complejos de salud, es necesario delimitar y asignar correctamente su rol en la controversia como garante de este derecho humano fundamental.

En este rumbo, cabe destacar la orientación de la jurisprudencia y el valor de sus precedentes. Así, la propia Corte Suprema ha dicho que: "Las obligaciones sanitarias de la autoridad local no implican desconocer el deber de coordinación con el Estado Nacional -mediante el Ministerio de Salud- el que debe acudir en forma subsidiaria, de manera de no frustrar los derechos de la amparista; de no ser así, las leyes sancionadas en la materia no dejarían de ser sino enfáticas enumeraciones programáticas vacías de operatividad" (CSJN, Fallos 329:2552).

Al respecto, es ilustrativo el voto en disidencia de la Dra. Carmen Argibay, en los términos siguientes: "Opino que en el presente caso existe materia federal para ser examinada por esta Corte, pues se ha controvertido la interpretación que adjudica responsabilidad al Estado Nacional como garante del derecho a la preservación de la salud de todos sus habitantes. Según el recurrente su obligación se limita a satisfacer las necesidades sanitarias de aquellas personas que no tienen cobertura alguna, razonamiento que colisiona con la hermenéutica efectuada por esta Corte en la causa A.186.XXXIV Asociación Benghalensis y otros c/ Ministerio de Salud y Acción Social - Estado Nacional s/ amparo ley 16.986" (Fallos: 323:1339) que le ha asignado responsabilidad subsidiaria sin efectuar distingos de ningún tipo. A mi entender, si bien es cierto que los jueces aclararon que sólo ante el incumplimiento de la condena por parte de la codemandada I.N.S.S.J.P. (PAMI) la amparista estará habilitada para ocurrir al Estado Nacional, no tratar en esta oportunidad los planteos que dedujo cierra la posibilidad de revisarlos en el futuro" (CSJN, "AD., M. de los A. c/ I.N.S.S.J.P. y/o E.N. s/ amparo", D. 809. XLII., 01/07/2008).

En el ámbito del Poder Judicial de la Provincia de Córdoba, resulta esclarecedor el voto reciente de la Dra. Inés del Carmen Ortiz de Gallardo, “...En este sentido, la Ley 24.901 ha creado un sistema de prestaciones básicas de atención integral a favor de las personas con discapacidad para brindarles asistencia integral, poniendo su cobertura en primer lugar a cargo de obras sociales, la Provincia de Córdoba y el Estado Nacional. Este razonamiento no implica afectar el principio del federalismo, ni pone en crisis las facultades reservadas por los gobiernos locales en la organización de su sistema de salud, que debe ser concebido en base a un federalismo de concertación en materia sanitaria, en el que las omisiones o dilaciones en adoptar las medidas legislativas y/o reglamentarias necesarias, no puede sostenerse como óbice para la efectiva vigencia de los derechos constitucionales fundamentales, dejando establecida la responsabilidad primaria del Estado Provincial y del Estado Nacional, en un supuesto como el de M.O.S. que es el primer caso de solicitud de cobertura planteado ante la APROSS a partir de una Enfermedad Poco Frecuente" (Auto 239/21, "S., M. E. y otro c/Administración Provincial del Seguro de Salud (A.P.R.O.S.S.) - Amparo (Ley 4915)", Cámara Contencioso Administrativa de Segunda Nominación de la Ciudad de Córdoba).

En consonancia con el perfil de un Estado afianzado en su rol y función de garante primario del derecho a la salud, el Dr. Horacio Rosatti, Vocal de la Corte Suprema de la Nación ha expuesto: "Complementariamente, no puede obviarse en esta instancia que el Estado Nacional ha asumido compromisos internacionales encaminados a promover y facilitar la vigencia de los derechos, sin que el federalismo constituya obstáculo para ello (Pacto Internacional de Derechos Económicos, Sociales y Culturales, arto 28; Convención 
Americana sobre Derechos' Humanos, arto 28.2 , no siendo razonable desligarse de los deberes asumidos so pretexto de la inactividad de otras entidades públicas o privadas, máxime cuando ellas participan de un mismo sistema sanitario y lo que se halla en juego es la rehabilitación integral del minusválido, que debe ser tutelada por todos los departamentos gubernamentales" (CSJN, Fallos: 323:3229, ya citado, considerando 21).

En sentido coincidente también se ha afirmado enfáticamente que: “En suma, el Estado es garante del derecho a la salud, cuya vulneración habilita a interponer la acción de amparo. El derecho a la salud no obliga al Estado a curar ni a lograr el completo bienestar, sino a brindar, en la medida de los recursos disponibles -decimos aquí parafraseando al Comité de Derechos Económicos, Sociales y Culturales de las Naciones Unidas en su Observación General N 14- las condiciones, prestaciones y bienes necesarios para lograr el más alto nivel posible de salud. Y ello ha de ser, como bien dice la Corte Suprema de Justicia de la Nación, mediante acciones positivas -y progresivas, agregamos aquí-, esto es, a través de un rol activo y no de mero espectador (fallos 321:1684; 323:1339; 323:3229; 324:3569, entre muchos otros)" (Urbina P., 2017).

Entonces, a la luz de estos valiosos precedentes corresponde destacar que el Estado, por mandato legal, tiene la trascendente encomienda de velar por la protección del derecho a la salud desde un enfoque del sistema de los derechos humanos. Los estándares jurídicos del control de constitucionalidad y de convencionalidad refuerzan lo antedicho.

No obstante, la señalada y especial vocación y función del Estado, "cuando esté de por medio la salud y con ella, el derecho a la vida de las personas, la labor jurisdiccional debe partir necesariamente de un pormenorizado análisis de las circunstancias vitales particulares y singulares que subyacen al planteo, en aras de dotar de equidad a la solución que se procure" (Palacio de Caeiro S., 2015).

De modo que, para hacer efectivos los derechos cuya protección se requiere, la función jurisdiccional no puede soslayar las circunstancias, contingencias y contornos de la controversia en materia de salud que se le presenta.

En esa sintonía, y proyectando estos conceptos al caso judicial bajo comentario (en el cual la cuestión a resolver concernía a una persona en situación de discapacidad), estamos en condiciones de afirmar que, tal como lo tiene dicho la doctrina, la experiencia de la discapacidad es singular para cada individuo, no solo porque la manifestación concreta de la enfermedad, desorden o lesión es única, sino porque tal condición de salud se encuentra necesariamente influida por una compleja combinación de factores (personales, emocionales, psicológicos, intelectuales, contexto social, físico y cultural) (Egea García, C. y Sarabia Sánchez, A. (2001).

Realizadas estas aclaraciones, y en torno a nuestro interrogante, podemos afirmar que la ineludible obligación del Estado y su responsabilidad en controversias de salud se delinea en todos los casos frente a un derecho fundamental como lo es el derecho a la salud. La salud, en cuanto bien individual y social a alcanzar, requiere necesariamente de la presencia del Estado porque consagra obligaciones a su cargo. Ambos conceptos conviven (o debieran hacerlo) en un razonado equilibrio.

Por otra parte, como fue antes esbozado, el cumplimiento de la obligación del Estado se tornaría más estricto ante ciertos límites, esto es, frente a situaciones de enfermedad de 
mayor gravedad y complejidad. Ese límite, en definitiva, entre el carácter principal o subsidiario de la responsabilidad del Estado en materia de salud transita por las situaciones de "extrema vulnerabilidad".

\section{Conclusiones.}

La obligación del Estado en materia de salud implica una tarea y un rol a desempeñar encomendada por la Constitución Nacional y por el bloque supraconstitucional con carácter imperativo.

El desarrollo del presente trabajo pretende ser tan solo una aproximación a los interrogantes formulados respecto de la obligación del Estado como garante del derecho a la salud, delineando los límites de su responsabilidad y el carácter en el cual resulta comprometido en este tipo especial de controversias judiciales.

Sin perjuicio de la postura a adoptar acerca del carácter primario o subsidiario con el cual el Estado debe responder, es claro que, en las reclamaciones basadas en la tutela del derecho a la salud, los operadores jurídicos, so pena de incurrir en arbitrariedad, deben fundamentar sus decisiones ponderando las concretas circunstancias del caso desde un enfoque protectorio de los derechos humanos que garantice los derechos de la tutela judicial efectiva y de la defensa en juicio.

Tal como pone de resalto la OMS que el enfoque de la salud basado en los derechos humanos ofrece estrategias y soluciones que permiten afrontar y corregir las desigualdades, las prácticas discriminatorias y las relaciones de poder injustas que suelen ser aspectos centrales de la inequidad en los resultados sanitarios (OMS, 2017).

Si aceptamos este estándar de interpretación estaremos en condiciones de desandar caminos más reales, pero no menos justos.

\section{REFERENCIAS BIBLIOGRÁFICAS}

- Egea García, C. y y Sarabia Sánchez, A. (2001); “Clasificaciones de la OMS sobre discapacidad", Servicio de Información sobre Discapacidad (SID) perteneciente a la Red Española de Información sobre Discapacidad. Disponible en: https://sid.usal.es/idocs/F8/ART6594/ clasificacion_oms.pdf

- Organización Mundial de la Salud (2017). Salud y derechos humanos. (Notas descriptivas). Ginebra. Suiza. Disponible en: https://www.who.int/es/news-room/fact-sheets/detail/human-rights-and-health

- Palacio de Caeiro S., (2015), Acción de amparo en Córdoba, Advocatus, Córdoba, pág. 560.

- Rodríguez Traversa, M. L., (2014); Amparo contra obras sociales: acerca de la improcedencia de demandar de manera directa al Estado Nacional, Sistema Argentino de Información Jurídica (SAIJ), Id. SAIJ: DACF140766.

- Tafani R. y Tafani M.L., (2010); Gestión y Gerencia Publica. Revista de Salud Pública, UNC, Disponible en: https://revistas.unc.edu.ar/index.php/RSD/article/view/7080

- Urbina, P. A. (2017), El derecho a la salud como obligación estatal, Ministerio de Salud, Presidencia de la Nación. Disponible en: https://salud.gob.ar/dels/entradas/el-derechola-salud-como-obligacion-estatal 